\title{
Aceite de oliva y prevención cardiovascular: más que una grasa
}

\author{
PABLO PÉREZ MARTÍNEZ \\ JOSÉ LÓPEZ MIRANDA \\ JAVIER DELGADO-LISTA \\ FERNANDO LÓPEZ-SEGURA \\ FRANCISCO PÉREZ JIMÉNEZ \\ Hospital Universitario Reina Sofía \\ Universidad de Córdoba
}

En la última década ha crecido el interés por la Dieta Mediterránea (DM), al vincular su consumo a una mayor longevidad y menor incidencia de enfermedades cardiovasculares, cáncer o deterioro cognitivo. La DM comparte muchas de las características de otras dietas saludables, como la vegetariana, la de los países latinos o las de las poblaciones orientales, pero el hecho de que tenga un alto contenido en grasa le da un interés especial, ya que aumenta su palatabilidad y explica su gran aceptación popular. Ahora bien, la mayor parte de su contenido graso procede de un solo alimento, el aceite de oliva, lo que explica que sea una dieta pobre en grasa saturada y rica en monoinsaturada (MONO), en especial en ácido oleico. Adicionalmente, su presencia en la dieta favorece que se consuman con más facilidad distintos productos vegetales, como las frutas, verduras, legumbres, hortalizas y cereales, alimentos con alto contenido en hidratos de carbono de baja carga glucémica y de gran potencialidad saludable. Gracias a ello los beneficios de la DM no se deben exclusivamente al aceite de oliva, sino al conjunto global de la dieta. Durante muchos siglos la producción de aceite de oliva se circunscribía a la cuenca del Mar Mediterráneo, por lo que una dieta de esas características sólo era posible en los países de su entorno. En los últimos años, y gracias a la moderna tecnología, se dispone para la nutrición humana de otros aceites, con una composición grasa parecida, como son los procedentes de ciertas semillas, con variedades altas en oleico, como el girasol, soja y colza. Ello ha generado un nuevo concepto de DM, de lo que son ejemplos la Dieta del Estudio de Lyon y la Dieta Indomediterránea, en los que el ácido oleico alimentario no procede predominantemente del aceite de oliva sino de distintas variedades de semillas. Sin embargo, esos aceites tienen una diferencia fundamental con el procedente de la aceituna, y es que este último es un zumo natural, que 
contiene cientos de microcomponentes no grasos, de gran potencialidad biológica, entre los que se incluyen la vitamina E, los carotenos, el escualeno, la clorofila y, en especial, los compuestos fenólicos. Dentro de ellos los más numerosos pertenecen a tres clases, los fenoles simples (tirosol e hidroxitirosol), los secoroides (la oleuropeina y sus formas conjugadas) y los lignanos. En contraposición con ello, los aceites procedentes de semillas han de ser refinados antes de su consumo, por lo que carecen de la gran mayoría de sus microcomponentes originales, con lo que son casi exclusivamente una fuente de grasa. Existe una variedad comercial de aceite procedente de la aceituna, el denominado comercialmente «aceite de oliva», que contiene un pequeño porcentaje de aceite virgen (5-10\%), que se añade al aceite de oliva previamente refinado, por lo que su riqueza en microcomponentes es menor que la de los aceites vírgenes, aunque sea superior a los de semilla. Finalmente, el aceite de orujo es originario de la aceituna pero totalmente refinado, por lo que es una buena fuente de MONO, con limitados microcomponentes del aceite virgen. Estas consideraciones son importantes porque en este trabajo discutiremos los beneficios de la dieta rica en aceite de oliva virgen, la DM genuina, cuyos efectos derivan no sólo de su contenido en grasa MONO sino del resto de sustancias potencialmente saludables. Por otra parte, desde el Estudio de los Siete Países y los conocidos trabajos de Keys, surgió un gran interés por clarificar el efecto de la grasa MONO sobre el metabolismo del colesterol. Posteriormente, en especial en la última década, se ha desarrollado un nuevo paradigma, con la demostración de que los efectos de la dieta van mucho más allá del colesterol e, incluso, de los factores de riesgo tradicionales, como discutiremos aquí y puede verse en recientes revisiones.

\section{ACEITE DE OLIVA Y FACTORES DE RIESGO CARDIOVASCULAR}

Los beneficios más conocidos de la dieta rica en MONO, sobre los niveles plasmáticos de colesterol, fueron los primeros que generaron interés por la DM. Como corolario de ello, el 1 de noviembre del 2004, la FDA (Food and Drug Administration) americana autorizó la difusión de mensajes saludables sobre el aceite de oliva, señalando que dos cucharaditas diarias (23 gramos) pueden reducir el riesgo coronario, cuando reemplaza a la misma cantidad de grasa saturada. Es de notar que el informe se apoyó en 73 ensayos clínicos, destacando cuatro de ellos por su especial calidad, siendo dos de ellos de nuestro propio grupo, uno de otro autor español y el cuarto de un grupo americano. Gracias a ello quedó establecido que el consumo de dicho alimento mantiene el colesterol HDL y reduce 
el colesterol LDL, cuando sustituye a una fuente de grasa saturada. Pero además, los MONO descienden los triglicéridos al sustituir a los hidratos de carbono, en el contexto de las dietas pobres en grasa. Puesto que este efecto sobre el colesterol LDL y HDL se ha demostrado con aceites ricos en MONO, cualquiera que fuera su origen, este efecto no es exclusivo del aceite de oliva sino que lo producen otros aceites refinados, ricos en ácido oleico. No obstante, hay datos experimentales que indican que los polifenoles de los aceites virgen y extravirgen podrían influir adicionalmente en el metabolismo lipídico, reduciendo la actividad de la HMG-CoA reductasa y modificando los niveles lipídicos. Hará falta realizar estudios en humanos para comprobar si estos efectos se reproducen en nuestra especie. Además de los efectos sobre el metabolismo lipídico tradicional, el aceite de oliva puede influenciar el metabolismo lipídico postprandial, como han mostrado recientes estudios.

La lipemia postprandial es la situación metabólica habitual en la que se encuentra el ser humano a lo largo del día, y se caracteriza por un aumento de los triglicéridos totales y de las lipoproteínas ricas en triglicéridos de origen intestinal y hepático. Diversos estudios sugieren que dichas partículas contribuyen al desarrollo de la arteriosclerosis. Por otra parte la lipemia postprandial está influenciada por el tipo de grasa presente en la dieta. Los quilomicrones que se originan tras la ingesta del aceite de oliva entran más rápidamente en la circulación y son aclarados de forma precoz que aquellos que se originan tras la ingesta de la grasa saturada o poliinsaturada, como el aceite de cártamo. El metabolismo acelerado de los quilomicrones otorgaría al aceite de oliva una característica menos aterogénica, incluso si la magnitud de la lipemia postprandial fuera similar a la originada por otros tipos de grasa. Por otra parte, diferentes tipos de aceites ricos en MONO pueden originar niveles similares de lípidos plasmáticos, en el estado basal y, sin embargo, la respuesta postprandial puede ser diferente. En este sentido, Abia et al. demostraron que el consumo del aceite de oliva virgen, comparado con el aceite de girasol alto en oleico, reduce la respuesta de las lipoproteínas ricas en triglicéridos durante el postprandio. Esto sugiere que existen otros factores en la composición del aceite de oliva que pueden ser responsables de estos efectos metabólicos. Un nuevo concepto, que actualmente está en estudio, es el de la influencia de la dieta habitual sobre la respuesta postprandial a la ingesta lipídica. Silva et al. compararon una dieta rica en grasa saturada (dieta control) frente a otras dos, con un contenido moderado o alto en MONO. Tras la intervención, las más ricas redujeron la producción de apoB-48, lo que sugiere que el tamaño de los quilomicrones se incrementa con dicha grasa, concluyendo que origina quilomicrones de mayor tamaño y potencialmente menos aterogénicos. 
Otro factor de riesgo, también muy vinculado a la dieta, es la presión arterial. Su posible modificación por el aceite de oliva es menos conocida que lo que sucede con el metabolismo lipídico, aunque desde hace años se ha sugerido en estudios observacionales y, últimamente, en la cohorte española del Proyecto SUN. Algunos ensayos clínicos, realizados recientemente en personas sanas, han profundizado igualmente en el beneficio de la DM, mostrando una reducción de los niveles tensionales, en comparación con dietas pobres en grasa, o ricas en saturada. Un efecto similar se ha constatado en diabéticos tipo 2 y, en un estudio muy interesante, Ferrara et al. refrendó el efecto beneficioso del aceite de oliva en pacientes hipertensos, al ver que, los que seguían una dieta rica en grasa poliinsaturada, requerían más tratamiento antihipertensivo que los que consumían aceite de oliva. También se ha demostrado que la ingesta isocalórica de este alimento, comparado con el de girasol, reduce la presión sistólica en ancianos hipertensos, lo que no se ha confirmado al comparar los aceites de oliva virgen y de soja, en pacientes hipertensos. Pero los datos más recientes y especialmente interesantes son los de Esposito et al. quienes mostraron, en un total de 180 pacientes con síndrome metabólico, que una DM se asociaba a una significativa reducción de la presión sistólica y diastólica a largo plazo (dos años), además de inducir otros muchos efectos antiinflamatorios y metabólicos, como luego comentaremos. Aunque en el modelo global de DM hay otros nutrientes que podrían reducir las cifras tensionales, el aporte tan importante de calorías que supone el aceite de oliva le da un protagonismo especial a este alimento. Más recientemente, en una cohorte griega perteneciente al estudio EPIC, que incluía a más de 20.000 personas, observaron que la adherencia a la DM se asociaba a cifras tensionales más bajas, siendo el aceite de oliva un predictor individual de dicho efecto. Pero también existen trabajos que sugieren que tal acción no tendría que ser exclusiva de la grasa MONO, sino de los microcomponentes del aceite de oliva virgen. En un ensayo clínico, realizado en mujeres hipertensas, se constató que el beneficio tensional se evidenciaba especialmente con este tipo de aceite, y no cuando se consume aceite de girasol alto en oleico. Aunque esos hallazgos no siempre se han confirmado en personas sanas, cabe la posibilidad de que los componentes no grasos pudieran tener un efecto hipotensor propio. Ello sugiere la observación de que el aceite de oliva, con elevado contenido en compuestos fenólicos, reducía la presión sistólica en pacientes hipertensos, con enfermedad coronaria, al compararlo con un aceite refinado del mismo origen. Tal vez tales efectos varían entre grupos de población, lo que puede limitar la generalización de los resultados, aunque el tema podría ser mucho más complejo. 
Otro factor de riesgo cardiovascular principal es la diabetes mellitus tipo 2. En un estudio ya clásico se demostró que una dieta rica en MONO (33\% del aporte calórico diario), redujo las necesidades de insulina en pacientes diabéticos, en comparación con otra rica en hidratos de carbono. Diferentes estudios posteriores parecían corroborar tales datos, y un metaanálisis mostró que la grasa MONO, comparada con los hidratos de carbono, mejoró tanto el perfil lipídico como el índice glucémico, sin diferencias en los niveles de insulina basales y en los de hemoglobina glicada. Desde entonces se han completado varios ensayos clínicos, con resultados dispares, al no confirmar siempre diferencias en el control glucémico, o en el perfil lipídico, de enfermos o de personas en alto riesgo de desarrollar diabetes tipo 2. En un trabajo posterior de nuestro grupo, en una población joven y sana, no se observaron diferencias en la sensibilidad periférica a la insulina tras la ingesta de una dieta rica en MONO, frente otra rica en hidratos de carbono. No hay pues datos concluyentes de que estos últimos aumenten la resistencia periférica a la insulina en personas normales. Otra cuestión sería lo que ocurre en personas con sensibilidad a la insulina disminuida, en las cuales la ingesta de hidratos de carbono incrementaría la oferta de glucosa, forzando la necesidad de hormona, lo que se traduciría en un empeoramiento del metabolismo de los hidratos de carbono. En tales casos la ingesta isocalórica de MONO mejoraría el metabolismo de la insulina. Otra cuestión diferente es lo que sucede con los saturados, como recientemente, en el estudio KANWU, han demostrado Vessby et al. Al modificar la proporción de ácidos grasos, disminuyendo los saturados y aumentando los MONO, se mejora la sensibilidad a la hormona en personas sanas, aunque no se modificó su secreción. Por tanto, la ingesta de grasa saturada puede aumentar la resistencia a la insulina, mientras que la MONO la disminuye, estando condicionado el efecto de los hidratos de carbono a la sensibilidad previamente subyacente a la acción de la hormona.

\section{EFECTOS DEL ACEITE DE OLIVA SOBRE OTROS MECANISMOS DE LA ARTERIOSCLEROSIS}

A partir de los llamativos resultados del «Estudio del Corazón de Lyon», en que el beneficio cardiovascular quedaba sin explicar por la modificación de los factores de riesgo, se comenzó a pensar que la intervención dietética induce efectos mucho más complejos que la acción sobre las lipoproteínas. A partir de ahí se ha desarrollado una extensa investigación, confirmándose los múltiples efectos "pleiotrópicos» de la grasa MONO, como antes hemos apuntado. Pero además, existen datos que sugieren 
que, en el caso del aceite de oliva virgen, se producirían beneficios adicionales, dependientes también de sus componentes minoritarios, como seguidamente comentaremos.

\section{BENEFICIOS SOBRE LA HEMOSTASIA}

Aunque existe aún mucho que avanzar, este campo ha merecido un especial interés, habida cuenta de que en las personas de elevado riesgo cardiovascular podría existir una activación crónica de los mecanismos de la trombosis, condicionando lo que se podría calificar de un ambiente protrombótico. Hace años, Sirtori et al., observaron que una dieta rica en aceite de oliva, en personas sanas, disminuía la sensibilidad de las plaquetas al colágeno, mientras que el aceite de maíz aumentaba el umbral de agregabilidad al ácido araquidónico. Posteriormente se confirmó que una dieta rica en MONO, comparada con otra de elevado contenido en poliinsaturados, disminuía la excreción urinaria de 11-dehydro-tromboxano $\mathrm{B}_{2^{\prime}}$ un metabolito del $\mathrm{TXB}_{2^{\prime}}$ derivado a su vez del TXA $2_{2^{\prime}}$ producto con actividad proagregante, generado por la acción de la ciclooxigenasa en las plaquetas y el endotelio. Posteriores estudios han dado resultados confusos y paradójicos, que incluso podrían ser, con respecto a los MONO, desfavorables o similares a los de distintos ácidos grasos, con un incremento de la excreción urinaria de metabolitos del TXB2 en comparación con la grasa saturada. Son muchas las razones que podrían explicar resultados dispares, cuestión por otra parte habitual en los estudios de intervención, dada su gran complejidad y las dificultades propias de tales ensayos, heterogéneos en sus diseños, con peculiaridades propias de distintas poblaciones o con una gran variabilidad metodológica y experimental, incluido la potencial confusión derivada de los muchos alimentos o nutrientes que se consumen junto a la grasa en estudio. De todos modos, los trabajos mas recientes parecen confirmar los resultados iniciales, que apuntaban un beneficio de la ingesta de MONO sobre la coagulación y la fibrinolisis. En uno de ellos, a las 8 semanas de una dieta rica en aceite de colza y girasol, con alto contenido en oleico, se redujo la agregabilidad plaquetaria a tres agonistas (ácido araquidónico, colágeno y ADP), si bien a las 16 semanas sólo se mantenía el efecto sobre el último. Los mismos autores confirmaron esos datos en un cuidadoso estudio, con dos niveles de ingesta de grasa MONO ( $18 \%$ y $15 \%$ del aporte calórico global), al compararla con grasa saturada $(16 \%)$. Aunque el beneficio del consumo elevado fue mayor a corto plazo (8 semanas), el efecto de ambos niveles de ingesta se igualaba a la larga (16 semanas), manteniendo una disminución de la agregación inducida 
por el ácido araquidónico. Otra cuestión interesante es si la riqueza en componentes minoritarios, de los aceites utilizados, podrían inducir una influencia específica. En ese sentido, Visioli et al., observaron que $40 \mathrm{ml} /$ día de aceite de oliva virgen, rico en compuestos fenólicos, se acompañó a las 7 semanas de una disminución de los niveles plasmáticos de TXB2, frente a un aceite con un contenido más bajo en tales componentes. Más recientemente el mismo autor ha confirmado estos hallazgos, ahora con experimentos realizados durante la fase postprandial de la ingesta de dos aceites similares a los del anterior estudio. Ello concuerda perfectamente con los datos encontrados "in vitro", con células endoteliales de cordón umbilical, que secretaban menos TXB2 cuando se exponían a partículas postprandiales obtenidas tras el consumo de aceite de oliva rico en microcomponentes. Finalmente, un factor circulante que favorece la adherencia de las plaquetas al endotelio denudado, el Factor von Willebrand, podría reducirse con dietas ricas en aceite de oliva, aunque el significado biológico de estos hechos no se conozca con precisión.

De los factores de la hemostasia, uno de los que ha suscitado un interés más especial es el Factor VII (FVII) ya que, junto a los de la fibrinolisis, podría tener un papel central en el desarrollo de enfermedad coronaria. En los primeros estudios se estableció que su actividad en ayunas se incrementa con la ingesta habitual de cualquier tipo de grasa, de modo que el ácido palmítico aumentaría los niveles de FVII coagulante (FVIIc) mientras que la grasa MONO elevaría los del FVII activado (FVIIa). En un estudio realizado con ácido oleico se observó un efecto posiblemente ligado al sexo, ya que sólo las mujeres tendrían niveles más bajos, en comparación con la grasa saturada. El efecto favorable también se ha confirmado con una dieta rica en MONO, procedentes de colza, en pacientes hipertrigliceridemicos, y en una población en la que se comparó un modelo de DM, frente a otro pobre en grasa o rico en grasa saturada, con menores niveles de FVIIa con la primera. La posibilidad de que haya un efecto propio de la grasa MONO, sobre los niveles de FVIIc, se refuerza porque sus niveles descendieron con una dieta rica en aceite girasol alto en oleico, en personas sanas. En ese mismo sentido Larsen et al. no observaron diferencias ni en FVIIa ni en FVIIc, al comparar entre si varios aceites de semilla, enriquecidos en MONO (girasol, oliva y colza), aunque en un estudio postprandial el incremento de FVIIa fue menor tras el aceite de oliva virgen, sugiriendo que sus micronutrientes podrían amortiguar la activación del FVII inducida por la grasa. Un hecho interesante, que se ha puesto en evidencia recientemente, es que la activación postprandial de FVII, tras la ingesta aguda de grasa, podría depender de la dieta habitual que mantenga la persona, de modo que el consumo crónico del aceite de oliva evitaría la activación tras una 
ingesta aguda de grasa. Parecidos resultados se observaron con una dieta prolongada rica en MONO (18\% de aporte energético), comparada con otra más pobre en dicho tipo de grasa $(15 \%)$, procedente de aceites refinados. Ese hecho confirmado por otros autores, se ha explicado porque la dieta rica en MONO favorece que el número de quilomicrones postprandiales sea inferior, atenuando la activación del factor indicado.

Otro mecanismo clave e importante de la hemostasia es la fibrinolisis, crucial para la reabsorción del trombo. Su actividad depende de la acción del Activador Tisular del Plasminógeno (tPA) gracias al cual se genera la plasmina, enzima que hidroliza la fibrina recién formada y evita su crecimiento. Su mecanismo regulador más importante depende de los niveles plasmáticos del Inhibidor del Activador Tisular del Plasminógeno tipo 1 (PAI-1), los cuales se incrementan con dietas ricas en ácido palmítico. Sin embargo, una DM, rica en aceite de oliva, redujo sus niveles, en comparación con otra pobre en grasa. Existen sin embargo datos discrepantes, como un estudio posterior, utilizando aceite refinado de semillas, mientras que nuevamente el aceite de oliva virgen, en pacientes hipertensos, redujo los niveles plasmáticos de PAI-1, en comparación con aceite de soja. Nuevos trabajos, con dietas ricas en aceite de oliva, confirmaban de nuevo su efecto beneficioso, lo que lleva a pensar que pudiera existir una acción específica, de sus micronutrientes, sobre la fibrinolisis. Menos definitivos son aún los datos sobre el fibrinógeno, tal vez porque al ser un marcador inflamatorio sus niveles podrían depender principalmente de la inflamación subyacente. En un estudio en el que los participantes consumieron una dieta rica en MONO, procedente de aceites refinados, se incrementaron sus niveles mientras que la DM los redujo, en paralelo con otros parámetros inflamatorios. Serán necesarios más estudios para definir estos efectos.

\section{ENDOTELIO Y ACEITE DE OLIVA}

La alteración inicial, que precede al desarrollo de arteriosclerosis, es la afectación endotelial, lo que condiciona que sus células expresen en la superficie moléculas, que favorecen la adhesión de las células mononucleares circulantes. De este modo se iniciaría el proceso inflamatorio que conduce a la pérdida de sus propias funciones. Entre ellas es especialmente relevante la respuesta vasodilatadora dependiente del óxido nítrico, y la capacidad de reducir el riesgo trombogénico, a la cual nos hemos referido. El mecanismo celular que media la expresión de los genes de respuesta inflamatoria, tanto en el endotelio como en las demás células que participan 
en la inflamación de la pared vascular, depende de los factores de transcripción, entre los que es especialmente conocido el $\mathrm{NF}_{\mathrm{K}} \mathrm{B}$, mediador que es sensible a los cambios oxidativos. En este campo tiene especial interés la demostración de que la suplementación con ácido oleico, a un medio de cultivo de células endoteliales, reduce la activación transcripcional del citado factor en dichas células, de un modo similar a como lo hace un ácido graso omega-3, el alfa linolénico, y al contrario del efecto proinflamatorio del ácido linoleico. Este hecho es congruente con la demostración previa de Carluccio et al., quienes observaron, también en un modelo de cultivo de células endoteliales, que la incorporación de ácido oleico a los lípidos de la membrana celular disminuyó la expresión de la molécula de adhesión VCAM-1, la primera que se expresa en la superficie de las células, cuando se inicia su activación. Esta acción antiinflamatoria de los MONO también explica que, el enriquecimiento de las partículas de LDL con ácido oleico, durante el consumo de distintos tipos de dieta, disminuyera su capacidad para inducir quimiotaxis y adhesión de los monocitos. En un paso más, nosotros hemos observado recientemente que la ingesta de aceite de oliva reducía la expresión de $\mathrm{NF}_{\mathrm{K}} \mathrm{B}$ en células mononucleares, obtenidas durante la fase postprandial de personas sanas, de un modo similar a lo que también se observaba con la ingesta de ácido linolénico, y al contrario del efecto proinflamatorio de la grasa saturada. Pero además, en ese mismo estudio, el aceite de oliva redujo el nivel plasmático de otra molécula de adhesión, el ICAM-1. Este efecto antiinflamatorio se ha observado, en el caso de la DM, tanto en estudios observacionales como de intervención. Especialmente relevante es el trabajo, antes mencionado, y publicado por Esposito et al. al observar, en un ensayo clínico sobre 180 pacientes con síndrome metabólico, de dos años de duración, que la DM redujo la prevalencia de dicho síndrome, mejorando la sensibilidad a la insulina y disminuyendo los niveles de PCR e interleucinas 6, 7, 18.

Aunque aún nos movemos en un terreno bastante especulativo, el efecto antiinflamatorio del aceite de oliva habría que considerarlo en el contexto de la inflamación que se produce durante la ingesta de dietas de alta energía, capaces de promover una superproducción de especies reactivas de oxígeno y de inducir cambios en la Fracción 3 del complemento, con la consiguiente puesta en marcha de la disfunción endotelial, adhesión de leucocitos a la pared y consiguiente emigración al espacio subendotelial. Existen datos sugestivos de que las grasas con capacidad antioxidante o estabilizadoras de las membranas podrían proteger a las células endoteliales. En este contexto, los MONO tendrían un menor efecto prooxidante, evitando la activación de las células de la pared vascular. Esta capacidad se conoce desde hace años, cuando se demostró que las LDL aumentan 
su resistencia a la oxidación cuando la dieta tiene un mayor contenido en dicho tipo de grasa. Pero además, en el caso del aceite de oliva y por el efecto añadido de sus microcomponentes, su capacidad para amortiguar el ambiente prooxidante sería mayor, como se ha demostrado en los últimos años. De tales microcomponentes, la fracción probablemente más implicada, en dicha protección, son los compuestos fenólicos, entre los que predominan la oleuropeina, una aglicona en cuya hidrólisis se genera tirosol e hidroxitirosol, que en su forma libre y en sus formas secoroides y conjugadas representan el $80 \%$ de los compuestos fenólicos del aceite de oliva virgen. Estos productos se absorben en el intestino humano, de manera dosis dependiente, y en estudios experimentales han mostrado propiedades antioxidantes, actividad quimiopreventiva y capacidad para mejorar la función endotelial, disminuyendo la expresión de moléculas de adhesión celular, aumentando la disponibilidad de óxido nítrico y neutralizando los radicales libres intracelulares. Además, también pueden modificar la hemostasia, inhibiendo la agregación plaquetaria y mostrando propiedades antitrombóticas, tanto en estudios experimentales como de intervención humana. Se ha observado que la ingesta de aceite de oliva virgen, rico en dichos componentes, aumenta el contenido fenólico total de las LDL, por lo que serían susceptibles a ejercer su acción en la íntima arterial, donde se desarrolla la oxidación de las LDL. Por otra parte, existen estudios experimentales indicativos de que estos compuestos disminuyen in vitro la oxidación de las LDL, aunque el efecto antioxidante se detecta también in vivo, con un volumen de ingesta de aceite de oliva que entra dentro del consumo habitual. Por lo tanto la DM podría modular el estrés oxidativo celular, modificando las lipoproteínas y regulando a la baja los mediadores inflamatorios, amortiguando la activación de los genes implicados en la inflamación, probablemente a través del $\mathrm{NF}_{\mathrm{K}} \mathrm{B}$ u otros factores de trascripción. La riqueza en microcomponentes, del aceite de oliva virgen, es muy elevada y aún no conocemos el efecto de la mayoría de ellos, siendo posible incluso que actúen por un efecto directo, similar al de los antiinflamatorios no esteroideos, inhibiendo al sistema de las ciclooxigenasas, sin tener que mediar su acción por la acción antioxidante.

Como antes indicamos, existen evidencias de que la capacidad vasodilatadora del endotelio, dependiente de la biodisponibilidad endotelial de óxido nítrico, es uno de los mecanismos que primero se altera cuando dicha estructura se lesiona. Este fenómeno es tan precoz que incluso se detecta en presencia de un factor de riesgo aislado, sin necesidad de que existe una alteración anatómica demostrable de la pared arterial. El interés de este fenómeno se justifica porque dicha alteración tiene carácter predictivo del riesgo de sufrir episodios coronarios, además de que se dispone de 
métodos no cruentos para su detección, aunque no estén validados para la toma de decisiones en la clínica. En los últimos años se ha demostrado que distintos nutrientes, en especial los de mayor capacidad antioxidante, el ácido fólico, la grasa omega-3 o alimentos como el vino o el té, pueden mejorar la respuesta vasodilatadora endotelial. El efecto vasodilatador de los MONO es menos conocido, ya que los estudios iniciales mostraron que la ingesta de cualquier tipo de grasa empeoraría la respuesta vasodilatadora, corrigiéndose con la administración simultánea de antioxidantes, como vitamina E y C o una ensalada con vinagre. En un estudio de nuestro grupo observamos que un modelo de DM, en pacientes con hipercolesterolemia moderada, fue capaz de incrementar la dilatación dependiente del endotelio, respecto a una dieta rica en grasa saturada. Además, de un modo concordante, disminuyeron las cifras plasmáticas de P-selectina. Este estudio tiene el interés de que la dieta era rica en grasa, procedente en su mayoría del aceite de oliva virgen, desestimando la idea de que una ingesta elevada de grasa sería perjudicial. Para definir el posible papel de los MONO, y de los microcomponentes que existen en dicho alimento, hemos llevado a cabo un estudio, en pacientes con hipercolesterolemia, en el que observamos que la respuesta vasodilatadora, dependiente del endotelio, está condicionada por su riqueza en compuestos fenólicos. Pero además el aceite de oliva virgen indujo una mayor biodisponibilidad de óxido nítrico, reduciendo los niveles de marcadores de oxidación, como lipoperóxidos y F2- isosprostanos.

En resumen, la DM es un modelo de alimentación con abundante contenido en grasa MONO, capaz de inducir un amplio abanico de efectos biológicos sobre el sistema cardiovascular. Sin embargo, los estudios mas recientes están definiendo gradualmente que hay algunos beneficios que dependen o se potencian con la ingesta de aceite de oliva rico en microcomponentes, como sucede con el aceite de oliva virgen. Por tanto este alimento tendría un doble beneficio, de una parte vinculado a su tipo de grasa y de otra dependiendo de su concentración en micronutrientes. Gracias a ello, el predominio de dicho alimento en nuestra dieta, favorecerá un mejor perfil lipídico, un ambiente menos protrombótico, tendrá un efecto antioxidante y antiinflamatorio, con mayor capacidad para proteger el endotelio. En el futuro, la aplicación de los enfoques disponibles de genómica funcional, las técnicas proteómicas, la metabonómica y la biología de sistemas, en su conjunto, facilitarán una comprensión más integral de sus efectos saludables. En cualquier caso parece evidente que la grasa fundamental de la DM debe ser el aceite de oliva y no otra fuente grasa, aunque tengan un elevado contenido en ácido oleico. 


\section{BIBLIOGRAFÍA}

De Lorgeril M, Renaud S, Mamelle N, Salen P, Martin JL, Monjaud I Guidollet J, Touboul P, Delaye J. Mediterranean alpha-linolenic acid-rich diet in secondary prevention of coronary heart disease. Lancet 1994;343:1454-59.

Fuentes, F, Jopez-Miranda, J, SÁnchez, E, SÁnchez, G, Paez, J, Paz-Rojas, E., Marin C, Gomez P, Jimenez-Pereperez J, Ordovas JM, PereZ-Jimenez F. Mediterranean and low-fat diets improve endothelial function in hypercholesterolemic men. Ann Intern Med 2001;134:1115-19.

Hu F. The Mediterranean diet and mortality. Olive oil and beyond. N Engl J Med 2003;348:2595-2596.

Jansen, S., Lopez-Miranda, J., CAstro, P., Lopez-Segura, F., MArin, C., OrdoVAs, J.M., Paz E, Jimenez-Pereperez J, Fuentes F, Perez-Jimenez F. Low-fat and high-monounsaturated fatty acid diets decrease plasma cholesterol ester transfer protein concentrations in young, healthy, normolipemic men. Am J Clin Nutr 2000;72:36-41 .

Mata P., Garrido J.A., Ordovas J.M., Blazquez E., Alvarez-Sala L.A., Rubio M.J., Alonso R, DE OYA M. Effect of dietary monounsaturated fatty acids on plasma lipoproteins and apolipoproteins in women. Am J Clin Nut 1992;56:77-83

PÉREZ-Jiménez F, LÓPEZ-Miranda J, MATA P. Protective effect of dietary monounsaturated fat on arteriosclerosis: beyond cholesterol. Atherosclerosis 2002; 163:385-98.

Pérez-Jiménez F, LóPez-Miranda J, MATA P. Protective effect of dietary monounsaturated fat on arteriosclerosis: beyond cholesterol. Atherosclerosis 2002; 163:385-98.

Pérez-Jiménez F, Fuentes F, Fernández de la Puebla R, López-Miranda J. Efectos pleiotrópicos de la grasa de la dieta sobre el riesgo cardiovascular. Clin Invest Arterioscler 2003;15: 27-32.

Pérez-Martínez P, López-Miranda J, López-Segura F, Delgado J, Pérez-Jiménez F. Aceite de oliva y prevención cardiovascular: más que una grasa. 2006; 18 : 195-205. 